\title{
The high-energy environment in the super-Earth system CoRoT-7
}

\author{
K. Poppenhaeger ${ }^{1}$, S. Czesla ${ }^{1}$, S. Schröter ${ }^{1}$, S. Lalitha ${ }^{1}$, V. Kashyap ${ }^{2}$, and J. H. M. M. Schmitt ${ }^{1}$ \\ ${ }^{1}$ Hamburger Sternwarte, Gojenbergsweg 112, 21029 Hamburg, Germany \\ e-mail: katja.poppenhaeger@hs . uni-hamburg.de \\ 2 Harvard-Smithsonian Center for Astrophysics, 60 Garden Street, Cambridge, MA 02138, USA
}

Received 23 November 2011 / Accepted 28 February 2012

\section{ABSTRACT}

\begin{abstract}
High-energy irradiation of exoplanets has been identified to be a key influence on the stability of these planets' atmospheres. So far, irradiation-driven mass-loss has been observed only in two Hot Jupiters, and the observational data remain even more sparse in the super-Earth regime. We present an investigation of the high-energy emission in the CoRoT-7 system, which hosts the first known transiting super-Earth. To characterize the high-energy XUV radiation field into which the rocky planets CoRoT-7b and CoRoT-7c are immersed, we analyzed a $25 \mathrm{ks} X M M$-Newton observation of the host star. Our analysis yields the first clear $(3.5 \sigma) \mathrm{X}$-ray detection of CoRoT-7. We determine a coronal temperature of $\approx 3 \mathrm{MK}$ and an X-ray luminosity of $3 \times 10^{28} \mathrm{erg} \mathrm{s}^{-1}$. The level of XUV irradiation on CoRoT-7b amounts to $\approx 37000 \mathrm{erg} \mathrm{cm}^{-2} \mathrm{~s}^{-1}$. Current theories for planetary evaporation can only provide an order-of-magnitude estimate for the planetary mass loss; assuming that CoRoT-7b has formed as a rocky planet, we estimate that CoRoT-7b evaporates at a rate of about $1.3 \times 10^{11} \mathrm{~g} \mathrm{~s}^{-1}$ and has lost $\approx 4-10$ earth masses in total.
\end{abstract}

Key words. stars: activity - planetary systems - planets and satellites: atmospheres - X-rays: stars - stars: coronae $\mathrm{X}$-rays: individuals: CoRoT-7

\section{Introduction}

After more than a decade of finding Jovian exoplanets, planetary research has entered a new stage heralded by the discovery of low-mass - possibly Earth-like - exoplanets. Léger et al. (2009) reported on the detection of the first known transiting superEarth, CoRoT-7b. In quick succession, intense radial-velocity follow-up revealed a second, CoRoT-7c (Queloz et al. 2009), and potentially a third planet, CoRoT-7d (Hatzes et al. 2010), making CoRoT-7 a compact super-Earth system.

CoRoT-7b orbits its host star every $0.85 \mathrm{~d}$, has a mass of $\approx 7.4 M_{\oplus}$, and a semi-major axis of 0.017 AU (Hatzes et al. 2011). CoRoT-7c orbits at a distance of $0.046 \mathrm{AU}$, has roughly twice CoRoT-7b's mass, and an orbital period of $3.7 \mathrm{~d}$ (Queloz et al. 2009). The spectral properties of the host star, CoRoT-7, have been analyzed before (Léger et al. 2009; Bruntt et al. 2010). CoRoT-7 has an effective temperature of $5250 \pm 60 \mathrm{~K}$, a surface gravity of $\log (g)=4.47 \pm 0.05$, and a metal overabundance of $[\mathrm{M} / \mathrm{H}]=0.12 \pm 0.06$ with an abundance pattern consistent with that of the Sun. Bruntt et al. (2010) concluded that CoRoT-7b orbits a main-sequence star of spectral type G8V-K0V.

The two super-Earths CoRoT-7b and CoRoT-7c cause RV amplitudes of only $\approx 5 \mathrm{~m} \mathrm{~s}^{-1}$. Accurate RV measurements in the CoRoT-7 system are made difficult because of the host star's activity; indeed, the analysis of Queloz et al. (2009) showed that the dominating RV signal can be attributed to stellar rotation and, thus, activity. Those authors found a mean $\log \left(R_{H K}^{\prime}\right)$ index of -4.612 and a $2 \%$ variation in phase with the $23 \mathrm{~d}$ stellar rotation period. This result is in line with the optical variability observed in the CoRoT light-curve (Léger et al. 2009). Pinpointing the exact mass of CoRoT-7b has proven to be difficult because of the fairly high activity of the host star. The mass estimates of various authors differ by several Earth masses, see for example
Queloz et al. (2009), Hatzes et al. (2010), and Ferraz-Mello et al. (2011); here we use the mass determination from Hatzes et al. (2011) of $M_{\mathrm{p}}=7.4 M_{\oplus}$, yielding a mean planetary density of $\rho_{\mathrm{p}}=10.4 \mathrm{~g} \mathrm{~cm}^{-3}$.

Interestingly, the known super-Earths differ drastically in their physical properties. For example, the Earth-like density of $7-10 \mathrm{~g} \mathrm{~cm}^{-3}$ found for CoRoT-7b (Bruntt et al. 2010; Hatzes et al. 2011) is in sharp contrast to findings for GJ 1214b, which shows a density of only $1.9 \pm 0.4 \mathrm{~g} \mathrm{~cm}^{-3}$, suggesting the existence of a gaseous envelope (Rogers \& Seager 2010; Nettelmann et al. 2011). One possible source of these differences is the highenergy irradiation from the planets' host stars, which has been identified as the main driver of planetary mass-loss and is therefore an important factor for their evolution (e.g., Sanz-Forcada et al. 2010). Observational evidence for ongoing evaporation has been found for two transiting Hot Jupiters: HD 209458b (VidalMadjar et al. 2003) and HD 189733b (Lecavelier Des Etangs et al. 2010). The accumulated effect of stellar high-energy emission could have left fingerprints on today's planetary population (Sanz-Forcada et al. 2010). For CoRoT-7b, Valencia et al. (2010) showed that the accumulated planetary mass-loss may be as high as $100 M_{\oplus}$ if the planet initially hosted a massive hydrogenhelium envelope and ca. 3-4 $M_{\oplus}$ if the inital planetary density was similar to today's value.

To shed light on the possible atmospheric evaporation of CoRoT-7b, we present here the first measurement of the highenergy emission of the super-Earth host-star CoRoT-7.

\section{Data analysis}

CoRoT-7 was observed with XMM-Newton for approximately $25 \mathrm{ks}$ on September 22, 2010 (ObsID 0652640201, PI V. Kashyap). The observation does not cover any planetary transit. 


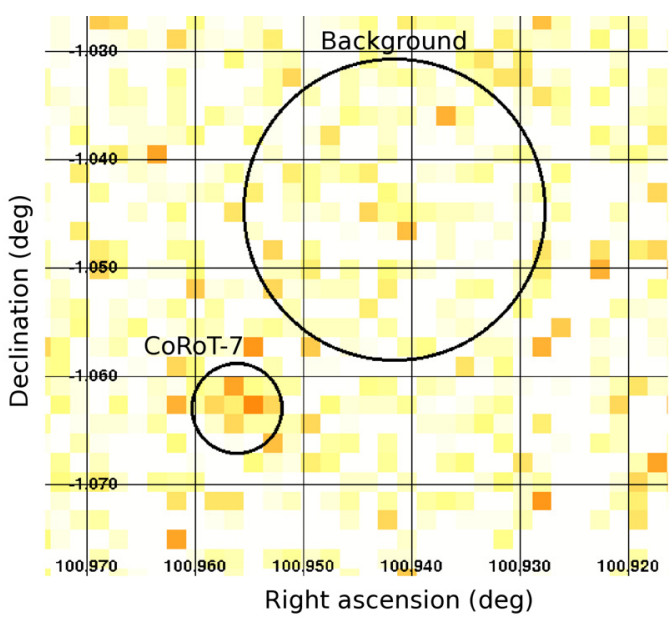

Fig. 1. Soft X-ray image of CoRoT-7, merged data from XMM-Newton's MOS1, MOS2, and PN camera in the $0.2-2 \mathrm{keV}$ energy band.

For our analysis of the X-ray data we used SAS version 11.0 and followed standard routines for data reduction. The observation, carried out with the thin filter, is afflicted by strong background after the first $10 \mathrm{ks}$; the background contribution is particularly strong in the PN detector. In our analysis we did not disregard time intervals of strong background, as this would have meant losing most of the data, too. However, the EPIC background contributes strongest at energies above $2 \mathrm{keV}$ and can therefore be reduced by choosing an approriate low-energy range where moderately active stars have their strongest X-ray emission.

In Fig. 1 we show the soft X-ray image of CoRoT-7. We extracted the source signal from a circular region with $15^{\prime \prime}$ radius centered on the nominal, proper-motion corrected position of CoRoT-7 from SIMBAD; the background signal was measured in a source-free region close to CoRoT-7 and subtracted from the signal in the source region.

Our analysis was carried out in the soft energy band from $0.2-2 \mathrm{keV}$, as this is where stellar emission is typically strong for weakly to moderately active stars as CoRoT-7, see for example Telleschi et al. (2005). In this way, the strength of the background in relation to the source signal was reduced.

$X M M$-Newton's Optical Monitor (OM) was used in the fast mode with the UVW1 filter inserted during the observation. The UVW1 filter covers a wavelength range of ca. 240-360 nm. In Fig. 2, we show the OM light-curve with $200 \mathrm{~s}$ binning obtained from the raw data.

To obtain a distance estimate from interstellar absorptionfeatures, we analyzed 46 optical VLT-UVES spectra of CoRoT7 obtained during five nights (Sep. 14, 2008, Dec. 28, 2009, Jan. 03, 2010, Jan. 07, 2010, Feb. 07, 2010; programs 081.C0413(C) and 384.C-0820(A)). The data were reduced using the ESO UVES-pipeline in version 4.4.8 (Ballester et al. 2000). These data have been analyzed before to study possible emission and absorption features of the planetary atmosphere, resulting in a nondetection and an upper limit of $2-6 \times 10^{-6} L_{*}$ for planetary emission in the Ca I, Ca II, and $\mathrm{NaD}$ lines (Guenther et al. 2011).

\section{Results}

To determine the X-ray luminosity of CoRoT-7 and the resulting evaporation rates, the distance to the system is crucial. Therefore, we start our discussion by deriving new and complementary distance estimates based on interstellar absorption and the Wilson-Bappu effect.

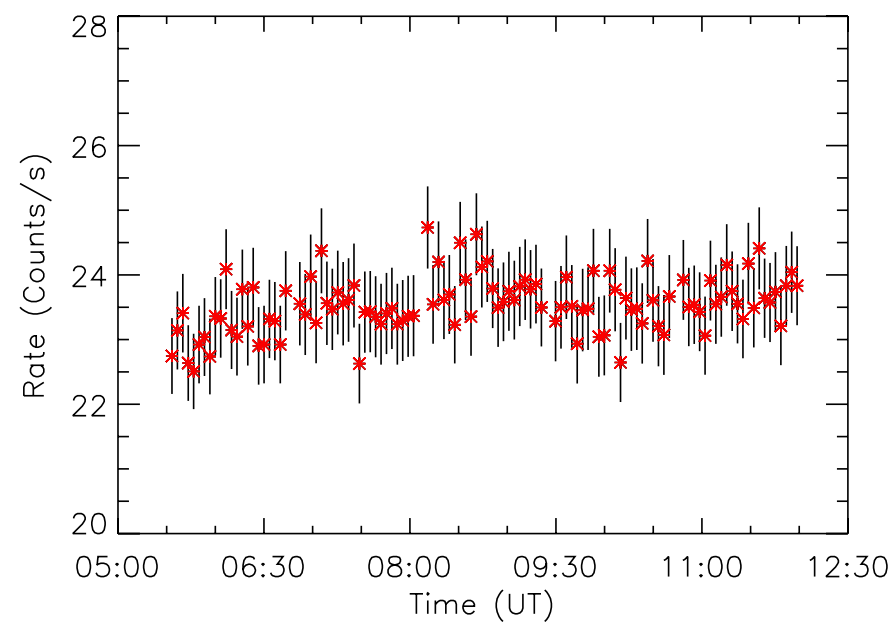

Fig. 2. UV light curve of CoRoT-7 recorded with XMM-Newton's Optical Monitor and rebinned to $200 \mathrm{~s}$ time resolution.

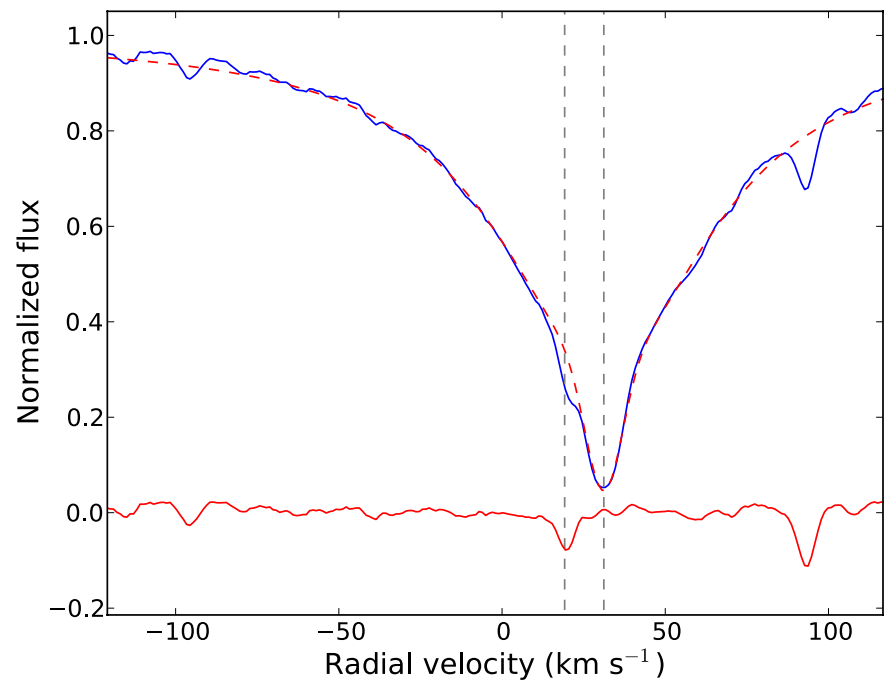

Fig. 3. Combined UVES spectra of CoRoT-7 showing the $\mathrm{Na}$ I $\mathrm{D}_{2}$ line and interstellar absorption. After subtracting a Voigt profile (dashed red) from the data (blue) the blueshifted absorption feature becomes visible in the residuals (solid red); the RV shift of the interstellar feature $\left(+19 \mathrm{~km} \mathrm{~s}^{-1}\right)$ and the CoRoT-7 system $\left(+31 \mathrm{~km} \mathrm{~s}^{-1}\right)$ are marked by vertical, dashed lines. The absorption lines in the outer parts of the line wing are due to Fe I.

\subsection{The distance to CoRoT-7}

In Fig. 3 we show an excerpt of the combined optical UVES spectra of CoRoT-7. In the blue wings of the Na I absorption-line doublet, we detected an additional, narrow absorption feature, which shows a relative displacement of $-12 \mathrm{~km} \mathrm{~s}^{-1}$ with respect to CoRoT-7 in radial velocity. This corresponds to a barycentric velocity shift of $+19 \mathrm{~km} \mathrm{~s}^{-1}$. Because the feature is visible in all spectra and shows no intrinsic variability, we attribute it to interstellar Na I absorption.

The equivalent widths (EWs) of the $\mathrm{Na} \mathrm{D}_{2}$ and $\mathrm{Na} \mathrm{D}_{1}$ features are $12.1 \pm 1.6 \mathrm{m \AA}$ and $6.8 \pm 1.8 \mathrm{m \AA}$. Using the line-ratio method (Strömgren 1948), we converted this measurement into a column density of $7 \times 10^{10} \mathrm{~cm}^{-2}$. Welsh et al. (2010) compiled a catalog of interstellar $\mathrm{Na}$ absorption lines and provide a map of the local interstellar medium, which we used to obtain a distance estimate from the absorption measured along the line-of-sight. Comparing the $\mathrm{Na}_{1,2}$ EWs with the catalog 
Table 1. X-ray net source counts of CoRoT-7 in the soft energy band $(0.2-2 \mathrm{keV})$.

\begin{tabular}{lccc}
\hline \hline & MOS 1+2 & PN & EPIC merged \\
\hline Source region & 183 & 567 & 750 \\
Background & 1389 & 5578 & 6967 \\
Net counts & 58 & 65 & 123 \\
Significance & $3.4 \sigma$ & $2.1 \sigma$ & $3.5 \sigma$ \\
\hline
\end{tabular}

Notes. Net counts obtained by subtracting the background signal after scaling it to the same area (factor 0.09).

data, we determined distance estimates of $160 \pm 80 \mathrm{pc}\left(\mathrm{D}_{2}\right)$ and $110 \pm 70 \mathrm{pc}\left(\mathrm{D}_{1}\right)$ for CoRoT-7; the errors were estimated as the standard deviation of the distances of stars with interstellar Na EWs in a $\pm 5 \mathrm{~m} \AA$ band around the measured EWs (cf., Schröter et al. 2011).

Using the Wilson-Bappu effect, Bruntt et al. (2010) obtained an absolute visual magnitude of $5.4 \pm 0.6 \mathrm{mag}$ for CoRoT-7. Given the apparent brightness of $V=11.7 \mathrm{mag}$, this translates into a distance of $180 \pm 50 \mathrm{pc}$ for CoRoT-7.

Although the results derived from $\mathrm{Na} \mathrm{D}_{2}$ and the photometric parallax favor a value of $\approx 170 \mathrm{pc}$, all estimates remain consistent with the distance of $150 \pm 20$ pc given by Léger et al. (2009). Therefore, we assume the same distance of $150 \mathrm{pc}$ in our analysis.

\subsection{X-ray and UV properties}

We report a clear X-ray detection of CoRoT-7. The merged EPIC image (MOS1, MOS2, and PN) shows an X-ray excess at the nominal position of CoRoT-7 (see Fig. 1). The detailed source and background count numbers are given in Table 1. From the merged data, we derived a detection significance of $3.5 \sigma$ in the soft energy band (0.2-2 keV).

Owing to the strong background signal a detailed analysis of the source's temporal variability and spectral properties remained impossible. To obtain an estimate of the coronal temperature despite this, we determined a hardness ratio from the collected source counts in the MOS1, MOS2, and PN detector. Using a soft band of $0.2-0.7 \mathrm{keV}$ and a hard band of $0.7-2.0 \mathrm{keV}$, we determined a hardness ratio of $H R=$ $-0.22_{-0.32}^{+0.25}$; the given errors denote the equal-tail $68 \%$ credibility interval.

We proceeded by calculating theoretical predictions for the hardness ratio assuming different coronal temperatures. The modeling was carried out using Xspec v12.0 and the result is shown in Fig. 4 along with our measurement. From this analysis, we can constrain the coronal temperature of CoRoT-7 to be between 2.5 and 3.7 MK, which is compatible with the coronal temperatures seen in moderately active stars such as the Sun at its maximum activity. Consequently, we assume a coronal temperature of $3 \mathrm{MK}$ to calculate CoRoT-7's X-ray luminosity.

At a stellar distance of $150 \mathrm{pc}$, interstellar hydrogen might produce noticeable absorption of the X-ray flux. According to Ferlet et al. (1985), the interstellar Nacolumn density is a suitable tracer for hydrogen. Our measurement of the sodium column density $N(\mathrm{Na})=7 \times 10^{10} \mathrm{~cm}^{-2}$ converts to a hydrogen column density of $N\left(\mathrm{H} \mathrm{I}+\mathrm{H}_{2}\right)=3 \times 10^{19} \mathrm{~cm}^{-2}$. Assuming a distance of $150 \mathrm{pc}$ to CoRoT-7 (see Sect. 3.1), this corresponds to a number density of about $0.1 \mathrm{~cm}^{-3}$ compatible with typical densities observed in the Local Cavity (Cox 2005).

Using WebPIMMS, we converted the mean count rate of a single XMM-Newton MOS instrument of $1.16 \times 10^{-3}$ counts s $^{-1}$

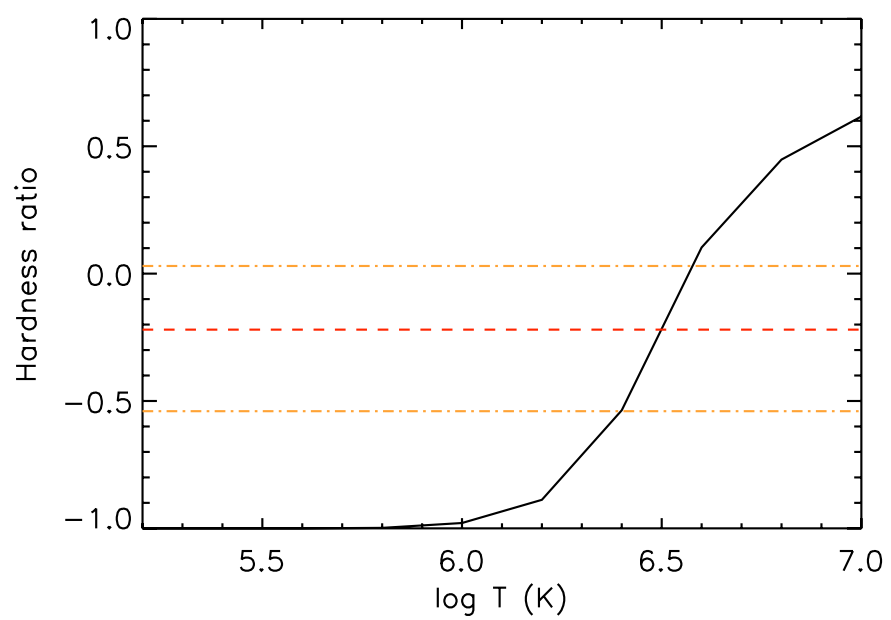

Fig. 4. Expected hardness ratio in XMM-Newton's MOS and PN detectors, depending on the mean coronal temperature (black); measured hardness ratio of CoRoT-7 (red dashed) shown with $68 \%$ credibility interval (orange dash-dotted).

into an unabsorbed X-ray flux of $1.0 \times 10^{-14} \mathrm{ergs} \mathrm{cm}^{-2} \mathrm{~s}^{-1}$ in the $0.2-2 \mathrm{keV}$ energy band, taking into account the encircled energy fraction of $68 \%$ for our $15^{\prime \prime}$ extraction region. Given a distance of $150 \mathrm{pc}$, this translates into an X-ray luminosity of $3 \times 10^{28} \mathrm{erg} \mathrm{s}^{-1}$. This is well in line with previous estimates derived from chromospheric $\mathrm{Ca}$ II $\mathrm{H}$ and $\mathrm{K}$ line emission, which yielded a predicted X-ray luminosity of $\approx 2 \times 10^{28} \mathrm{erg} \mathrm{s}^{-1}$ (Poppenhaeger \& Schmitt 2011). For a star of spectral type late $\mathrm{G}$ to early K, CoRoT-7 displays a typical moderate level of coronal activity, which is also observed in stars with solar-like activity cycles (Hempelmann et al. 2006; Favata et al. 2008): with a bolometric correction of -0.437 (Flower 1996), we derived a bolometric luminosity of $\log L_{\mathrm{bol}}=33.3$ and, therefore, an X-ray activity indicator, $\log L_{\mathrm{X}} / L_{\mathrm{bol}}$, of -4.8 .

The UV light-curve seen with the OM does not show strong flares (see Fig. 2). To determine the level and slope of the light curve, we fitted it with a linear model with free offset and gradient and determined errors using a Markov-Chain Monte-Carlo (MCMC) analysis. The mean count-rate amounts to 23.5(23.423.6) counts $\mathrm{s}^{-1}$, where the $95 \%$ credibility interval is given in parenthesis. Using the count-to-energy conversion factors for a star of spectral type $\mathrm{K} 0$ from the XMM-Newton handbook, we calculated a flux of $9 \times 10^{-15} \mathrm{erg} \mathrm{cm}^{-2} \mathrm{~s}^{-1}$ in the covered band of 240-360 nm. Combining this with a distance of $150 \mathrm{pc}$, we obtain a luminosity of $2.4 \times 10^{28} \mathrm{erg} \mathrm{s}^{-1}$ in the same band.

According to our analysis, the slope amounts to $8(1.8-14) \times 10^{-2}$ counts $^{-1}$, indicating a slight increase in UV luminosity during the observation, which may be due to intrinsic short-term variability induced by active regions or rotational modulation. Although a temporal analysis of the X-ray flux was not possible, the lack of pronounced UV variability indicates that it can be interpreted as quiescent emission.

\section{Discussion: evaporation of CoRoT-7b and CoRoT-7c}

Although an X-ray luminosity of $\approx 3 \times 10^{28} \mathrm{erg} \mathrm{cm}^{-2} \mathrm{~s}^{-1}$ does not place CoRoT-7 among the very active stars, the extreme proximity of its planets exposes them to high levels of high-energy irradiation.

Given semi-major axes of $0.017 \mathrm{AU}$ and $0.046 \mathrm{AU}$ for CoRoT-7b and CoRoT-7c (Queloz et al. 2009), we calculated 
X-ray fluxes of $f_{\mathrm{X}, \mathrm{b}}=3.7 \times 10^{4} \mathrm{erg} \mathrm{cm}^{-2} \mathrm{~s}^{-1}$ and $f_{\mathrm{X}, \mathrm{c}}=5 \times$ $10^{3} \mathrm{erg} \mathrm{cm}^{-2} \mathrm{~s}^{-1}$ at the orbital distances of the planets. Assuming a solar X-ray luminosity of $10^{27} \mathrm{erg} \mathrm{s}^{-1}$, these fluxes exceed the solar X-ray flux on Earth by factors of 100000 and 14000 .

We now derive an order-of-magnitude estimate for the amount of mass that has been evaporated from CoRoT-7b due to the stellar high-energy emission. Planetary evaporation is thought to be mainly driven by X-ray and EUV irradiation. The process is hydrodynamical (Tian et al. 2005; Murray-Clay et al. 2009) and is much more efficient than pure Jeans escape. The approach has been refined for expanded absorption radii and mass-loss effects through Roche lobe overflow (Lammer et al. 2003; Erkaev et al. 2007). However, there are still considerable uncertainties about the dynamics of the process. In addition, we assume here that CoRoT-7b has formed as a rocky planet and that the orbital distance is stable; there are other calculations that consider planetary migration as well as CoRoT-7b being the solid core of an evaporated gaseous planet, which consequently derive very different mass loss histories (Jackson et al. 2010). Here, we apply the same formula as used by Sanz-Forcada et al. (2011) and Valencia et al. (2010). The latter authors argue that the mass-loss rates, originally estimated for gaseous planets, can in principle remain true also for rocky planets, because the rate of sublimation in close vicinity to the star should be able to counterbalances the mass-loss:

$\dot{M}=\frac{3 \epsilon F_{\mathrm{XUV}}}{4 G \rho_{\mathrm{p}} K}$,

where $F_{\mathrm{XUV}}$ is the incident XUV flux at the planetary orbit, $G$ is the gravitational constant, $\rho_{\mathrm{p}}$ the mean planetary density, which we assume to be $10.4 \mathrm{~g} \mathrm{~cm}^{-3}$ according to Hatzes et al. (2011), and $\epsilon$ is a factor to account for heating efficiency of the planetary atmosphere, with $\epsilon=1$ denoting that all incident energy is converted into particle escape. Several authors chose $\epsilon=0.4$ (Valencia et al. 2010; Jackson et al. 2010), inspired by observations of the evaporating Hot Jupiter HD 209458b, and we follow their approach. However, this can only be a rough estimate of the true heating efficiency, which cannot be stated more precisely without detailed models of the dynamics of the exoplanetary atmosphere. $K$ is a factor for taking into account effects from mass loss through Roche lobe overflow; we neglect these effects by assuming $K=1$. This is justified because the approximate Roche lobe radius (Eggleton 1983) is about three times today's radius of CoRoT-7b. If the planetary mass had been three times as large initially, the Roche lobe radius would still have exceeded the initial planetary radius, as modeled by Seager et al. (2007) for rocky planets, by a factor of approximately two. Roche-lobe influenced mass loss was therefore likely insignificant for the planetary evolution.

Because we have not measured CoRoT-7's EUV flux, we use a relation between X-ray (0.1-2.5 keV) and EUV (100-920 ^) flux (Sanz-Forcada et al. 2011):

$\log L_{\mathrm{EUV}}=(4.80 \pm 1.99)+(0.860 \pm 0.073) \log L_{\mathrm{X}}$.

We measured the X-ray luminosity in the $0.2-2 \mathrm{keV}$ band to be $3 \times 10^{28} \mathrm{erg} \mathrm{s}^{-1}$; we used WebPIMMS to extrapolate this to the required energy band of $0.1-2.5 \mathrm{keV}$, the result being $3.3 \times 10^{28} \mathrm{erg} \mathrm{s}^{-1}$. This leads to an EUV luminosity of $L_{\mathrm{EUV}}=2.1 \times 10^{29} \mathrm{erg} \mathrm{s}^{-1}$, and thus a combined XUV (X-ray and EUV) luminosity of $L_{\mathrm{XUV}}=2.4 \times 10^{29} \mathrm{erg} \mathrm{s}^{-1}$ of CoRoT-7, which is about ten times higher than the X-ray luminosity alone. Substituting this number into Eq. (1) yields an estimate of $1.3 \times 10^{11} \mathrm{~g} \mathrm{~s}^{-1}$ for CoRoT-7b's current mass-loss rate.
This number is higher than the one derived by Valencia et al. (2010), who estimated the XUV flux purely from an age-activity relation for solar-like stars given by Ribas et al. (2005), since no X-ray detection of the star was available. CoRoT-7 has a slightly sub-solar mass, being of spectral type G8-K0; however, for an order-of-magnitude estimate of the mass loss, the difference in activity evolution of $\mathrm{G}$ and early $\mathrm{K}$ stars is small (Lammer et al. 2009) and can be neglected. Valencia et al. (2010) estimate the XUV luminosity as $5 \times 10^{28} \mathrm{erg} \mathrm{s}^{-1}$. Assuming a lower planetary density than in this work, $4-8 \mathrm{~g} \mathrm{~cm}^{-3}$, they derived a lower mass loss rate of $0.5-1 \times 10^{11} \mathrm{~g} \mathrm{~s}^{-1}$.

For CoRoT-7c, we estimate that the mass-loss rate should be about an order of magnitude lower than for CoRoT-7b due to the weaker irradiation. Because this planet does, however, not transit, its radius - and thus density - remains unknown.

Planetary mass-loss rates have been measured for two Hot Jupiters so far by using the transit depth in the hydrogen Ly $\alpha$ line as an indicator for extended and escaping planetary atmospheres. Vidal-Madjar et al. (2003) reported a lower limit for the mass loss rate of HD $209458 \mathrm{~b}$ of $\sim 10^{10} \mathrm{~g} \mathrm{~s}^{-1}$. Similarly, Lecavelier Des Etangs et al. (2010) derived a mass loss rate between $10^{9}$ and $10^{11} \mathrm{~g} \mathrm{~s}^{-1}$ for HD $189733 \mathrm{~b}$. The orders of magnitude are compatible with our calculations, even if these observational results were obtained for gaseous, not rocky planets.

Based on our mass-loss rate determination for CoRoT-7b, we can derive an order-of-magnitude estimate for the total planetary mass-loss over time. The possible stellar age range for CoRoT-7 is given by Léger et al. (2009) as 1.2-2.3 Gyr; we adopt here an age of 1.5 Gyr. Along the lines of Valencia et al. (2010) we assume a constant density of CoRoT-7b. Likewise, for the stellar activity evolution, we assume that the combined X-ray and EUV luminosity increases at younger stellar ages $\tau$ by a factor of $\left(\tau / \tau_{*}\right)^{-1.23}$ with $\tau_{*}$ being the current stellar age in Gyr (Ribas et al. 2005), and that the activity remains at a constant level for ages younger than 0.1 Gyr. Inserting the time-variable XUV flux into Eq. (1) and integrating over 1.5 Gyr yields a total mass loss of ca. $6 M_{\oplus}$. This is higher than the result of Valencia et al. (2010) of 3-4 $M_{\oplus}$, who used a lower stellar XUV flux and lower planetary density. If we consider the uncertainty in the age determination of CoRoT-7, our estimated mass loss range extends to 4-10 $M_{\oplus}$. Given the current uncertainties in the analytical models for planetary evaporation, we consider the possible range for the true mass loss of CoRoT-7b to be even wider.

\section{Conclusion}

We report the first X-ray detection of CoRoT-7, host star to at least two close-in super-Earths. After veryfying the distance estimate of $150 \mathrm{pc}$ of Léger et al. (2009), we converted the X-ray flux of $10^{-14} \mathrm{erg} \mathrm{cm}^{-2} \mathrm{~s}^{-1}$ measured with XMM-Newton into a luminosity of $3 \times 10^{28} \mathrm{erg} \mathrm{s}^{-1}(0.2-2 \mathrm{keV})$.

An X-ray activity indicator of $\log L_{\mathrm{X}} / L_{\mathrm{bol}}=-4.8$, combined with a likely coronal temperature of $\approx 3 \mathrm{MK}$, characterizes CoRoT-7 as a moderately active star. Employing an analytical model for planetary evaporation - with the caveat that many aspects of this evaporation are not yet understood -, the combined X-ray and EUV irradiation can be converted into an order-of-magnitude estimate of the planetary mass loss. We derive a current mass loss rate of $1.3 \times 10^{11} \mathrm{~g} \mathrm{~s}^{-1}$ for the closest planet, CoRoT-7b. Assuming that the planet was formed as a rock and integrating the likely activity history of the CoRoT7 system, we estimate that CoRoT-7b has suffered a total mass loss of $\approx 4-10$ Earth masses. 
K. Poppenhaeger et al.: The high-energy environment in the super-Earth system CoRoT-7

Acknowledgements. K.P. and S.L. acknowledge funding from the German Research Foundation (DFG) via Graduiertenkolleg 1351. S.C. and S.S. acknowledge financial support from DLR grant 50OR0703.

\section{References}

Ballester, P., Modigliani, A., Boitquin, O., et al. 2000, The Messenger, 101, 31 Bruntt, H., Deleuil, M., Fridlund, M., et al. 2010, A\&A, 519, A51

Cox, D. P. 2005, ARA\&A, 43, 337

Eggleton, P. P. 1983, ApJ, 268, 368

Erkaev, N. V., Kulikov, Y. N., Lammer, H., et al. 2007, A\&A, 472, 329

Favata, F., Micela, G., Orlando, S., et al. 2008, A\&A, 490, 1121

Ferlet, R., Vidal-Madjar, A., \& Gry, C. 1985, ApJ, 298, 838

Ferraz-Mello, S., Tadeu Dos Santos, M., Beaugé, C., Michtchenko, T. A., \& Rodríguez, A. 2011, A\&A, 531, A161

Flower, P. J. 1996, ApJ, 469, 355

Guenther, E. W., Cabrera, J., Erikson, A., et al. 2011, A\&A, 525, A24

Hatzes, A. P., Dvorak, R., Wuchterl, G., et al. 2010, A\&A, 520, A93

Hatzes, A. P., Fridlund, M., Nachmani, G., et al. 2011, ApJ, 743, 75

Hempelmann, A., Robrade, J., Schmitt, J. H. M. M., et al. 2006, A\&A, 460, 261

Jackson, B., Miller, N., Barnes, R., et al. 2010, MNRAS, 407, 910

Lammer, H., Selsis, F., Ribas, I., et al. 2003, ApJ, 598, L121
Lammer, H., Odert, P., Leitzinger, M., et al. 2009, A\&A, 506, 399

Lecavelier Des Etangs, A., Ehrenreich, D., Vidal-Madjar, A., et al. 2010, A\&A, 514, A72

Léger, A., Rouan, D., Schneider, J., et al. 2009, A\&A, 506, 287

Murray-Clay, R. A., Chiang, E. I., \& Murray, N. 2009, ApJ, 693, 23

Nettelmann, N., Fortney, J. J., Kramm, U., \& Redmer, R. 2011, ApJ, 733, 2

Poppenhaeger, K., \& Schmitt, J. H. M. M. 2011, Astron. Nachr., 332, 1052

Queloz, D., Bouchy, F., Moutou, C., et al. 2009, A\&A, 506, 303

Ribas, I., Guinan, E. F., Güdel, M., \& Audard, M. 2005, ApJ, 622, 680

Rogers, L. A., \& Seager, S. 2010, ApJ, 716, 1208

Sanz-Forcada, J., Ribas, I., Micela, G., et al. 2010, A\&A, 511, L8

Sanz-Forcada, J., Micela, G., Ribas, I., et al. 2011, A\&A, 532, A6

Schröter, S., Czesla, S., Wolter, U., et al. 2011, A\&A, 532, A3

Seager, S., Kuchner, M., Hier-Majumder, C. A., \& Militzer, B. 2007, ApJ, 669, 1279

Strömgren, B. 1948, ApJ, 108, 242

Telleschi, A., Güdel, M., Briggs, K., et al. 2005, ApJ, 622, 653

Tian, F., Toon, O. B., Pavlov, A. A., \& De Sterck, H. 2005, ApJ, 621, 1049

Valencia, D., Ikoma, M., Guillot, T., \& Nettelmann, N. 2010, A\&A, 516, A20

Vidal-Madjar, A., Lecavelier des Etangs, A., Désert, J., et al. 2003, Nature, 422, 143

Welsh, B. Y., Lallement, R., Vergely, J., \& Raimond, S. 2010, A\&A, 510, A54 